\title{
Las pruebas periciales: Tipologías y relación con las distintas causas judiciales penales
}

\author{
Miguel Ángel Soria \& Inma Armadans \\ Universidad de Barcelona
}

\begin{abstract}
RESUMEN
Se analizan 236 expedientes judiciales procedentes de las Secciones Penales de la Audiencia Provincial de Barcelona con la finalidad de establecer la relación entre el tipo de prueba pericial forense solicitada y el delito imputado.

Se analizaron un total de 204 periciales y tras una evaluación piloto se estructuró la recogida de información en función de 13 variables.

Los resultados muestran la existencia de un escaso volumen de pruebas periciales solicitadas en los distintos expedientes analizados. En delitos contra la salud pública y el medio ambiente se pidieron pruebas documentales y biológico/químicas, mientras que las médicas lo fueron en delitos de lesiones, homicidios y agresiones sexuales. Las policiales se dirigieron casi exclusivamente a delitos contra la salud pública. Todas ellas fueron solicitadas principalmente de oficio.

Las pruebas periciales psicológicas representaron el $10 \%$ de las solicitadas y se aplicaron a casos de agresión sexual. La fuente peticionaria fueron las partes, analizándose: la capacidad intelectual del acusado, las secuelas psicológicas y la credibilidad del testimonio.
\end{abstract}

Palabras clave: Psicología jurídica, pruebas psicológicas, profesión psicológica, pruebas criminales, sistema criminal, testimonio pericial. 


\begin{abstract}
We analyzed 236 judicial expedients from the Criminal Sections of Audiencia Provincial de Barcelona and 26 of Jury Tribunal with the objective to establish the relationship between the evidence presented and the crime.

We analyzed 204 experts forensic evidences After a pilot study the information collect was made in 13 factors.

The results show a low level of forensic evidences presented in court in the different judicial criminal expedients analyzed. In crimes against the public health and against the environment are based in documents and biological/ chemical evidence, but in crimes of physical aggression, homicides and sexual aggression are the medical evidence. The police experts are demanded always for the Tribunal and majority demanded in the biological / chemical evidence and psychiatrics, but the psychological evidence are solicited by the accusation or the defense of accused.

The psychological evidence represented a $10 \%$ of all and was applied majoritary in processes of sexual aggression sexual and studied: IQ, psychological sequels and testimony credibility.
\end{abstract}

Key words: Legal psychology, psychological evidence, professional psychology, criminal evidence, criminal system, expert testimony.

\title{
Introducción.
}

En el seno de la psicología jurídica se ha configurado un ámbito específico de estudio relativo a la toma de decisiones jurídicas, tanto de tribunales profesionales como de jurados legos. Dentro del primer grupo existen en la actualidad numerosas investigaciones que ponen de manifiesto una gran disparidad en las decisiones judiciales tanto en la decisión de encarcelar (Partdridge y Eldridge, 1974; Diamond y Zeisel, 1975; Arce, Tortosa y Alfaro 2003) como en la longitud de la pena (Sutton, 1978).

Los intentos de explicación de esa disparidad apuntan a la trascendencia tanto de variables legales como extralegales (Soria, 1998). Respecto al primer conjunto de factores, Kapardis (1985) encontró cómo los de mayor relevancia en la explicación de la variabilidad de las sentencias la recencia de la última condena, el historial delictivo, el tipo de acusación, la interacción pasada con el sistema de justicia, la recomendación de sentencia emitida por el funcionario supervisor de la libertad condicional o la provocación por parte de la víctima del delito. En la misma línea, Arce, Fariña, Novo y Seijo (2001) encontraron como un factor de orden legal como la estimación de la credibilidad de los distintos testimonios y el valor atribuido a de cada prueba en la 
decisión judicial explicaría la mayoría de la varianza de la disparidad encontrada en la sentencia impuesta.

Respecto a las variables extralegales, si bien su número es muy extenso, las más estudiadas por su influencia sobre el contenido de la sentencia hacen referencia al papel desempeñado por el propio agente decisor, la orientación socio-penal de la pena impuesta (rehabilitativo o utilitario) como factores principales que generan decisiones judiciales dispares (Sobral y Prieto, 1994).

Otro factor extralegal a tener en cuenta habitualmente estudiado es el género del agente decisor. Diversos estudios desarrollados destacaron como el sexo de los jueces incidían diferencialmente ante decisiones relativas a delitos de violación (Arce, Fariña y Fraga, 2000), pero otros estudios no encontraron dichas diferencias (Arce, Fariña, Novo y Seijó, 1999). La conclusión más probable es que la variable género puede ser una fuente de sesgos, especialmente en algunos tipos delictivos (violencia doméstica, agresiones sexuales) (Wikler, 1989; Schafran, 1987; Arce, Fariña y Fraga, 2000). Pero también en dinámicas propias de la Sala de Justicia (Breton, 1986; Riger, Foster-Fishman, NelsonKuna y Curran, 1995). Este segundo efecto se debería a un sesgo instrumental, al adecuar la actuación de los abogados a un tipo de estrategia judicial preconcebida (Kiesler, 1975; Riger, Foster-Fishman, Nelson-Kuna y Curran, 1995).

Finalmente, una última línea de trabajo de orden extralegal en España, utilizando el estudio de sentencias de archivo, ha puesto de manifiesto como están determinadas principalmente por la comisión de sesgos sistemáticos de juicio durante el proceso decisional de los órganos judiciales (Garrido y Herrero, 1995; Arce, Fariña y Novo, 1996, 2002). Tradicionalmente en el campo de la psicología de las sentencias se ha utilizado el concepto de "anclaje", entendido como la superposición de la condena impuesta por el juez teniendo en cuenta prioritariamente la petición del Ministerio Fiscal (Arce, Fariña y Novo, 1996, 2002). Ello se encontró en un nivel muy elevado en delitos contra la libertad sexual $(81.75 \%)$ pero fue significativamente menor en crímenes de violación (Garrido y Herrero, 1995, 1997). En síntesis el anclaje interacciona específicamente con el tipo de delito juzgado. 
Previamente a estos hallazgos, ya se habían formulado diversos modelos de decisión judicial con un substrato explicativo fundamentado en la asunción de sesgos y heurísticos (Fitzmaurice y Pease, 1986; Lawrence, 1984; Michon y Pakes, 1995; Saks y Kidd, 1986), por ello la petición de un determinado tipo de pruebas forenses u otros por las distintas partes y el propio órgano judicial puede implicar un sesgo motivacional inicial que conduciría a una percepción determinada del órgano juzgador y afectando ulteriormente a su decisión.

En dicho sentido consideramos que una sentencia judicial, cómo todo juicio social, implica distintos modelos explicativos de los mismos (Garrido, 1993; Martin, 1988). En primer lugar las decisiones de los distintos tribunales se basan en un conjunto de competencias estructuradas jerárquicamente y por funciones y secundariamente los distintos agentes jurídicos (jueces, abogados, fiscales, etc.) tienden a solicitar aquellas pruebas en función de sus propios intereses profesionales, sus conocimientos técnicos, etc., pero además el análisis e interpretación de sus resultados se produce nuevamente según sus propios recursos y habilidades.

Siguiendo dicha concepción Garzón (1989) hablaba de tres niveles de análisis: organizacional, técnico-profesional, e ideológico-filosófico. Es más, algunos estudios indican cómo el tipo de delito, las pruebas solicitadas, el sexo del autor, de la víctima, etc. juegan un papel fundamental en la imposición de la sentencia judicial (Champion, 1988; Freckelton, 1994; Harrel, 1981; MacMartin, 2000 y Walsh, 1984). De forma más específica en una investigación precedente se analizaban las sentencia reales impuestas por jueces en la Audiencia Provincial de Barcelona, observándose cómo los jueces a la hora de realizarlas consideraban en quinto lugar las pruebas practicadas, en el séptimo la consistencia de las pruebas y en el octavo las circunstancias del hecho (Hernández y Soria, 1994).

Por todo ello en el presente estudio se pretende conocer la relación entre el tipo de delito imputado y las pruebas solicitadas por las distintas partes o el órgano decidor situándose en el nivel técnico-profesional (Garzón, 1989). 


\section{Método.}

\section{Muestra}

Se analizaron 236 expedientes judiciales consecutivos de las Secciones Penales de la Audiencia Provincial de Barcelona del año 2002 con la finalidad de establecer la relación entre el tipo de prueba forense solicitada y el delito imputado.

\section{Procedimiento}

Para ello se sistematizaron 3 grandes grupos de pruebas:

- Las declarativas. Pruebas aportadas al proceso penal efectuadas por personas implicadas directa o indirectamente en los hechos juzgado (acusado y testigos) y que se fundamenta en su testificación en la vista oral.

- Las periciales. Pruebas aportadas por profesionales expertos en la temática juzgada, bien sean a solicitud del Tribunal o de alguna de las partes.

- Las documentales. Pruebas aportadas en la fase documental del procedimiento pudiendo incluir múltiples aspectos, desde informes de tratamiento a atestados policiales, grabaciones de cámaras, etc. En consecuencia necesitan un soporte material (papel, video, audio, etc.) para ser reproducidos pudiendo ser realizados por profesionales o no, pero que no han sido citados como expertos en la causa.

En el presente artículo nos centraremos en el análisis de las pruebas periciales.

Tras una evaluación piloto sobre 40 sentencias de la Sección $7^{\mathrm{a}}$ la recogida de información se estructuró en función de 13 variables: sentencia, Sala, sexo del tribunal (o su porcentaje), sexo del acusado, sexo de la víctima, tipo de delito, tipos de pruebas aportadas, número total de pruebas aportadas, dirección de la prueba, número de testimonios en la causa, sentencia judicial, grado de aceptación de las pruebas y solicitud de la prueba.

\section{Diseño}

Las pruebas periciales se agruparon en 5 grandes tipos:

a) Pruebas biológicas-químicas: informes toxicológicos; ADN, sangre, orina, fluidos corporales, análisis de fibras, vidrio, etc. 
b) Pruebas policiales: dactiloscópica, grafística, balística, alcoholemia, inspección ocular, etc.

c) Pruebas médico-forenses: anatomía patológica, antropología forense, informes de lesiones, etc.

d) Pruebas de psiquiatría forense: estado mental del acusado, las secuelas de la víctima, etc.

e) Pruebas de psicología forense: estado mental del acusado, credibilidad del testimonio, capacidad intelectual, secuelas psicológicas, secuelas neuropsicológicas, etc.

En el caso de los dos últimos tipos de pruebas debido a que podía existir un mismo objetivo pericial valorativo en algunos temas se adjudico a un tipo $u$ otro de prueba psicológica o psiquiátrica según dos condiciones: la tipología de profesional responsable de la prueba concreta aportada y en segundo término cuando se utilizó de forma clara y precisa métodos, técnicas e interpretaciones propias de dicha ciencia.

Los datos obtenidos se analizaron estadísticamente mediante el paquete informático SPSS+.

\section{Resultados.}

Del conjunto de 236 expedientes judiciales analizados se obtuvieron un total de 744 pruebas solicitadas, de las cuales 435 fueron de tipo declarativo (58,5\%), 105 documentales $(14,1 \%)$ y 204 periciales $(27,4 \%)$. 
Tabla 1. Pruebas declarativas y documentales.

\begin{tabular}{|l|c|c|}
\hline Declarativas & $\mathrm{N}$ & $\%$ \\
\hline Acusado/s & 232 & 31.18 \\
\hline Testigo/s & 203 & 27.28 \\
\hline Total declarativas & 435 & 58.5 \\
\hline Documentales & \multicolumn{2}{|l|}{} \\
\hline Atestado policial & 58 & 7.79 \\
\hline Otras pruebas documentales & 47 & 6.31 \\
\hline Total documentales & 105 & 14.1 \\
\hline Periciales & \multicolumn{2}{|l}{} \\
\hline Total periciales & 204 & 27.4 \\
\hline TOTAL PRUEBAS & $\mathbf{7 4 4}$ & $\mathbf{1 0 0}$ \\
\hline
\end{tabular}

Si analizamos las 204 pruebas periciales solicitadas por las distintas Salas Penales de la Audiencia Provincial de Barcelona, la mayoría procedían de la medicina forense (35.8\%) y de los estudios bioquímicos (30.9\%), seguidas de las psiquiátricas (12.7\%) y en cuarto lugar las psicológicas (10.3\%). Analizando los distintos tipos de delitos y las tipologías de pruebas solicitadas observamos como los delitos sexuales tienen un mayor volumen $(\mathrm{N}=51)$ y variedad tipológica en el tipo de pruebas abordadas por los Tribunales, siendo fundamentalmente utilizadas las ciencias médica y psicológica (33.3\% en ambos casos). Los delitos de homicidio provocaron esencialmente la demanda de peritaje procedentes de la medicina forense $(63.9 \%)$ y psiquiatría $(16.7 \%)$. En los delitos contra la salud pública destacaron las pruebas de base biológica $(66,7 \%)$ y las policiales $(21.4 \%)$.

Los delitos de lesiones provocan una demanda casi exclusiva de pruebas periciales propias de la medicina forense $(97 \%)$. 
Cuando observamos los delitos de recursos naturales destaca que la mayor parte de las pruebas proceda de la psiquiatría $(60 \%)$ pero su número total es muy escaso $(\mathrm{N}=10)$.

En delitos contra el medio ambiente la casi totalidad de las pruebas procedían de la ciencia biología (96\%).

Tabla 2. Tipos de delitos y pruebas periciales solicitadas.

\begin{tabular}{|c|c|c|c|c|c|c|c|c|c|c|c|c|}
\hline & \multicolumn{2}{|c|}{$\begin{array}{l}\text { Biológicas/ } \\
\text { químicas }\end{array}$} & \multicolumn{2}{|c|}{ Policiales } & \multicolumn{2}{|c|}{$\begin{array}{l}\text { Medicina } \\
\text { forense }\end{array}$} & \multicolumn{2}{|c|}{ Psiquiatría } & \multicolumn{2}{|c|}{ Psicología } & \multicolumn{2}{|c|}{ Totales } \\
\hline & $\mathrm{N}$ & $\%$ & $\mathrm{~N}$ & $\%$ & $\mathrm{~N}$ & $\%$ & $\mathrm{~N}$ & $\%$ & $\mathrm{~N}$ & $\%$ & $\mathrm{~N}$ & $\%$ \\
\hline Homicidio & 3 & 8.3 & 1 & 2.8 & 23 & 63.9 & 6 & 16.7 & 3 & 8.3 & 36 & 18.3 \\
\hline Lesiones & 0 & 0 & 0 & 0 & 32 & 97 & 1 & 3 & 0 & 0 & 33 & 16.7 \\
\hline Sexual & 5 & 9.9 & 2 & 3.9 & 17 & 33.3 & 10 & 19.6 & 17 & 33.3 & 51 & 25.9 \\
\hline Recursos naturales & 3 & 30 & 1 & 10 & 0 & 0 & 6 & 60 & 0 & 0 & 10 & 5.1 \\
\hline Medio ambiente & 24 & 96 & 1 & 4 & 0 & 0 & 0 & 0 & 0 & 0 & 25 & 12.7 \\
\hline Salud pública & 28 & 66.7 & 9 & 21.4 & 1 & 2.4 & 3 & 7.1 & 1 & 2.4 & 42 & 21.3 \\
\hline TOTAL & 63 & & 14 & & 73 & & 26 & & 21 & & 197 & 100 \\
\hline
\end{tabular}

Si analizamos el tipo de prueba y su utilización en los distintos tipos de delito observamos como las pruebas periciales bioquímicas son solicitadas principalmente en delitos contra la salud pública (13,7\%) y contra el medio ambiente (11.7\%).

Las pruebas periciales policiales son solicitadas principalmente en delitos contra la salud pública $(4.4 \%)$ y las procedentes de la medicina forense en delitos de lesiones $(15.7 \%)$ y homicidio (11.3\%).

Las pruebas periciales psiquiátricas se utilizan principalmente en agresiones sexuales (4.9\%), homicidios y contra los recursos naturales (2.9\% respectivamente). Finalmente, las psicológicas se concentran casi específicamente en agresiones sexuales (8.4\%) 
Sobre el conjunto de delitos juzgados las periciales médicas fueron las mayoritarias solicitadas $(35,8 \%)$, seguidas de las biológicas-químicas $(30,9 \%)$, las psiquiátricas $(12,8 \%)$, las psicológicas $(10,4 \%)$ y finalmente las policiales $(6,9 \%)$.

Tabla 3. Pruebas periciales solicitadas y tipo de delito.

BIOQUÍMICAS POLICIALES MÉDICAS PSIQUIÁTRICAS PSICOLÓGICAS

\begin{tabular}{|c|c|c|c|c|c|c|c|c|c|c|}
\hline Tipo de delito & $\mathrm{N}$ & $\%$ & $\mathrm{~N}$ & $\%$ & $\mathrm{~N}$ & $\%$ & $\mathrm{~N}$ & $\%$ & $\mathrm{~N}$ & $\%$ \\
\hline Homicidio & 3 & 1.5 & 1 & 0.5 & 23 & 11.3 & 6 & 2.9 & 3 & 1.5 \\
\hline Lesiones & 0 & 0 & 0 & 0 & 32 & 15.7 & 1 & 0.5 & 0 & 0 \\
\hline Sexual & 5 & 2.5 & 2 & 1 & 17 & 8.3 & 10 & 4.9 & 17 & 8.4 \\
\hline Recursos naturales & 3 & 1.5 & 1 & 0.5 & 0 & 0 & 6 & 2.9 & 0 & 0 \\
\hline Medio ambiente & 24 & 11.7 & 1 & 0.5 & 0 & 0 & 0 & 0 & 0 & 0 \\
\hline Salud pública & 28 & 13.7 & 9 & 4.4 & 1 & 0.5 & 3 & 1.5 & 1 & 0.5 \\
\hline Total pruebas solicitadas & 63 & 30.9 & 14 & 6.9 & 73 & 35.8 & 26 & 12.8 & 21 & 10.4 \\
\hline $\begin{array}{l}\text { Total pruebas no } \\
\text { solicitadas }\end{array}$ & 141 & 69.1 & 190 & 93.1 & 131 & 64.2 & 178 & 87.2 & 183 & 89.6 \\
\hline TOTAL PRUEBAS & 204 & 100 & 204 & 100 & 204 & 100 & 204 & 100 & 204 & 100 \\
\hline
\end{tabular}

Si analizamos la fuente de solicitud de la pericial aparece una clara diferencia en función del tipo de pruebas solicitadas y del delito cometido. Así la mayoría de pruebas biológicas/químicas se solicitaron de oficio (64\%), esencialmente informes toxicológicos (76.8\%). Entre las pruebas solicitadas de parte destacan el ADN (75\%) y los fluidos corporales $(62.5 \%)$.

Las pruebas periciales policiales se solicitan el $100 \%$ de oficio, al igual que las pruebas médico-forenses que se centran en informes de lesiones y con un volumen más bajo en la anatomía patológica (autopsia).

Las pruebas de la psiquiatría forense se solicitaron casi exclusivamente de oficio (93.5\%) y hacían especialmente referencia al estado mental del acusado (96.3\%). 
Finalmente las pruebas periciales psicológicas fueron solicitadas mayoritariamente por las partes (59.4\%) destacando la capacidad intelectual (66.7\%) las secuelas psicológicas y la credibilidad del testimonio (62.5 respectivamente). Cuando fueron solicitadas de oficio destaca la valoración del estado mental del acusado (46.2\%).

Tabla 4. Procedencia de la solicitud en las pruebas periciales biológicas y químicas.

\begin{tabular}{|lllllll|} 
& & OFICIO & \multicolumn{3}{c}{ PARTE } & \multicolumn{2}{l|}{ TOTAL } \\
& $\mathrm{N}$ & $\%$ & $\mathrm{~N}$ & $\%$ & $\mathrm{~N}$ & $\%$ \\
\hline Informes toxicológicos & 43 & 76.8 & 13 & 23.2 & 56 & 100 \\
\hline A.D.N. & 1 & 25 & 3 & 75 & 4 & 100 \\
\hline Fluidos corporales & 3 & 37.5 & 5 & 62.5 & 8 & 100 \\
\hline Análisis orgánicos & 10 & 47.6 & 11 & 54.4 & 21 & 100 \\
\hline Total & 57 & 64 & 32 & 36 & 89 & 100 \\
\hline
\end{tabular}

Tabla 5. Procedencia de la solicitud en las pruebas periciales psiquiátricas.

\begin{tabular}{|l|l|l|l|l|l|l|}
\hline & \multicolumn{2}{|l}{ OFICIO } & \multicolumn{2}{l|}{ PARTE } & \multicolumn{2}{l|}{ TOTAL } \\
\cline { 2 - 8 } & $\mathrm{N}$ & $\%$ & $\mathrm{~N}$ & $\%$ & $\mathrm{~N}$ & $\%$ \\
\hline Estado mental acusado & 26 & 96.3 & 1 & 3.7 & 27 & 100 \\
\hline Secuelas víctimas & 3 & 75 & 1 & 25 & 4 & 100 \\
\hline Total & 29 & 93.5 & 2 & 6.5 & 31 & 100 \\
\hline
\end{tabular}


Tabla 6. Procedencia de la solicitud en las pruebas psicológicas.

\begin{tabular}{|lllllll|}
\hline & \multicolumn{2}{l}{ OFICIO } & PARTE & TOTAL \\
& $\mathrm{N}$ & $\%$ & $\mathrm{~N}$ & $\%$ & $\mathrm{~N}$ & $\%$ \\
\hline Estado mental acusado & 6 & 46.2 & 7 & 53.8 & 13 & 100 \\
\hline Credibilidad testimonio & 3 & 37.5 & 5 & 62.5 & 8 & 100 \\
\hline Capacidad intelectual & 1 & 33.3 & 2 & 66.7 & 3 & 100 \\
\hline Secuelas psicológicas & 3 & 37.5 & 5 & 62.5 & 8 & 100 \\
\hline Total & 13 & 40.6 & 19 & 59.4 & 32 & 100 \\
\hline
\end{tabular}

\section{Discusión.}

Los resultados de la investigación señalan como la mayoría de pruebas forenses solicitadas por las Secciones Penales de la Audiencia Provincial de Barcelona proceden de la medicina forense seguidas de las biológicas/químicas, la psiquiatría y en cuarto lugar las psicológicas.

Las pruebas periciales de base biológico/químicas y las procedentes de la medicina forense son solicitadas en variados tipos de delitos, pero suelen adoptar una forma grupal, es decir, suelen solicitarse todas a la vez, a modo de pauta general. Pero su especificidad varía según el tipo de delito concreto perpetrado, así en delitos contra la salud pública, contra el medio ambiente y contra los recursos naturales se solicitan prioritariamente las biológicas/ químicas, mientras las médico-forenses fueron más solicitada en delitos de lesiones, homicidios y agresiones sexuales. Las policiales se utilizaron en delitos contra la salud pública.

Las periciales procedentes de la psiquiatría tienden a concentrarse en delitos contra los recursos naturales y las psicológicas lo hacen de forma casi exclusiva en agresiones sexuales, seguidas de aquellas relativas a homicidios y sus formas. 
Respecto a la fuente peticionaria de la prueba la mayoría se solicitan de oficio, no obstante existen diferencias significativas en función del tipo de prueba solicitada, así en las pruebas biológicas/ químicas el ADN y fluidos son solicitados mayoritariamente de parte frente a los informes toxicológicos que lo son de oficio.

En el caso de las pruebas psiquiátricas se realizan de oficio casi en su totalidad, pero en determinados delitos como homicidio, sexuales y de robo la petición tendía a formularse de parte.

En el caso de las pruebas psicológicas son demandadas prioritariamente por las partes, aunque su peso relativo fluctuaba según el tipo de prueba solicitada, siendo la de mayor porcentaje la evaluación de la capacidad intelectual del acusado.

En conjunto el estudio muestra como las partes y el órgano judicial en temas penales tienden a solicitar un escaso número de pruebas periciales en comparación con el número de expedientes judiciales tramitados $\mathrm{y}$ analizados. Esencialmente se demandaron pruebas biológicas y químicas, prioritariamente las periciales fueron solicitadas de oficio, específicamente se trataban de informes toxicológicos y se aportaron en delitos contra la salud pública.

Las pruebas periciales policiales son demandadas en un número bajo $\mathrm{y}$ fundamentalmente en delitos contra la salud pública. Las procedentes de la medicina forense son de tipo informes de lesiones y en delitos de lesiones. Las psiquiátricas se solicitan de oficio, prioritariamente sobre el estado mental del acusado y en delitos sexuales. Finalmente, las psicológicas representaron un 10\% del total, se aplicaron esencialmente en casos de agresión sexual, solicitándose mayoritariamente de oficio, contrariamente a todas las demás, se utilizaron principalmente en la peritación de tres temáticas: capacidad intelectual del acusado, secuelas psicológicas y credibilidad del testimonio.

Todo ello nos indica la existencia de un anclaje en la solicitud de pruebas y su objetivo. Así la fuente peticionaria varía según sus objetivos ulteriores, pero esta se fundamenta en los tres niveles de análisis establecidos por Garzón (1989) pudiendo hablarse de 
factores organizacionales, profesionales e ideológicos que actúan como sustrato decisional. Pero al mismo tiempo se puede relacionar con los procesos ulteriores de decisión judicial a los cuales tienden a dirigirse (Hernández y Soria, 1994).

Las limitaciones del estudio nos indican su relevancia a nivel de trabajo descriptivo, pero no permite elaborar estudios comparativos estadísticos, al menos en esta primera fase, siendo ello un elemento clave a superar en futuras investigaciones.

También debemos destacar como debe continuar investigándose la relevancia de las pruebas en la decisión judicial ulterior, especialmente en lo referente a la valoración según el tipo de disciplina científica que la aporte, de su tipología y de la fuente solicitante.

El déficit de estudios en esta área en nuestro país debe superarse mediante un mayor número de casos analizados y la posibilidad de realizar estudios con grupos comparativos estadísticos en distintas áreas geográficas y tipos de juzgados, pues consideramos que las variables socioculturales y la experiencial profesional pueden jugar un papel muy significativo en la modulación de los resultados. 


\section{Referencias}

Arce, R., Fariña, F., Novo, M., \& Seijó, D. (2001). Judges’ decision-making from within. En R. Roesch, R. R. Corrado, \& R. J. Dempster (Eds.), Psychology in the courts: International advances in knowledge (pp. 195-206). Nueva York: Routledge.

Arce, R., Fariña, F., Novo, M., \& Seijó, D. (1999). Judge's decision making from within. Comunicación presentada en el The First Joint Meeting of the American Psychology-Law Society and the European Association of Psychology and Law, Dublin.

Arce, R., Fariña, F., \& Fraga, A. (2000). Género y formación de juicios en un caso de violación. Psicothema, 12(4), 623-628.

Arce, R., Fariña, F., \& Novo, M. (1996, Agosto). Cognitive activity in sentencing. VI European Conference on Psychology and Law, Siena.

Arce, R., Fariña, F., \& Novo, M. (2002). Heurístico de anclaje en las decisiones judiciales. Psicothema, 14(1), 39-46

Arce, R.; Tortosa, F., \& Alfaro, E. (2003). Veredictos y análisis del contenido de las deliberaciones de los tribunales de jueces y jurados en el contexto jurídico español. Psicothema, 15(1), 127-135.

Breton, T. (1986). Empirical study finds gender bias in Rhode Island Courts. The National Law Journal, 8, 13.

Champion, D.J. (1988). Child sexual abusers and sentencing severity. Federal Probation, 52 (1), 53-57.

Diamond, S.S. \& Zeisel, H. (1975). Sentencing council: A study of sentence disparity and its reduction. University of Chicago Law Review, 43, 109-149.

Fitzmaurice, C. \& Pease, K. (1986). The psychology of judicial sentencing. Manchester: Manchester University Press.

Freckelton, I. (1994). Sentencing the substance dependent offender. Psychiatry psychology and law, 1(1), 11-22.

Garrido, E. (1993). La psicología de las sentencias judiciales en delitos sexuales. En M. Garcia (Comp.), Psicología social aplicada en los procesos jurídicos y políticos (pp15-32). Sevilla: Eudema

Garrido, E. \& Herrero, C. (1995). Fiscales y jueces: concordancia entre percepción y realidad. En E. Garrido, y C. Herrero (comp.), Psicología política, jurídica y ambiental (pp. 269-281). Salamanca: Eudema.

Garrido, E. \& Herrero, C. (1997). Influence of the prosecutor's plea on the judge's sentencing in sexual crimes: hypothesis of the theory of anchoring by Tversky y Kahneman. En S. Redondo, V. Garrido, J. Pérez, y R. Barberet (Eds.), Advances in psychology and law. International contributions (pp. 215-227) Berlín: Walter de Gruyter.

Garzón, A. (1989). Psicología y justicia. Valencia: Promolibro.

Harrel, W.A. (1981). The effects of alcohol use and offender remorsefulness on sentencing decisions. Journal of applied social psychology, 11 (1), 83-91.

Hernández, J.A. \& Soria, M.A. (1994). Psicologia de les sentencies. Barcelona: Generalitat de Catalunya (Centre d'Estudis Jurídics)

Kapardis, A. (1985). Sentencing by English magistrates as a human process. Nicosia, Chipre: Asselia Press.

Kiesler, S. B. (1975). Actuarial prejudice toward women and its implications. Journal of applied social psychology, 5, 201-216. 
Lawrence, J.A. (1984). Magisterial decision making: cognitive perspective and processes used in the courtroom information processing. En D.J. Müller; D.E. Blakman, \& A.J. Chapman (Eds.), Psychology and law (pp. 319-331). Chichester: John Wiley and Sons.

MacMartin, C. (2000). Discursive constructions of child sexual abuse: conduct, credibility and culpability in trial judgments. Dissertation abstracts international section, 61(3-B) pp: 1698.

Martin, A. (1988) La sentencia judicial desde un punto de vista psicosocial. Boletin de Michon, J.A. \& Pakes, F.J. (1995). Judicial decision making: a theoretical perspective. En R. Bull, y D. Carson (Eds.), Handbook of psychology in legal contexts (pp. 509-527). Chichester: John Wiley and Sons.

Partdridge, A. \& Eldridge, W. (1974). The second circuit sentencing study. Nueva York: Federal Judicial Center.

psicología, 20, 25-47.

Riger, S., Foster-Fishman, P., Nelson-Kuna, J., \& Curran, B. (1995). Gender bias in courtroom dynamics. Law and Human Behavior, 19(5), 465-480.

Saks, M.J. \& Kidd, R.F. (1986). Human processing information: trial by heuristics. En H.R. Arkes, \& R.H. Hammond (Eds.), Judgment and decision making. An interdisciplinary reader (pp. 213-242). Cambridge: Cambridge University Press.

Schafran, L. H. (1987). Documenting gender bias in the courts: The task force approach. Judicature, 70, 280-290.

Sobral, J. \& Prieto, A. (1994). Psicología y ley. Un examen de las decisiones judiciales. Madrid: Eudema.

Soria, M.A. (1998). Psicología y práctica jurídica. Barcelona: Ariel.

Sutton, L. (1978). Variations in federal criminal sentences: $A$ statistical assessment at the national level. Washington, D.C.: National Criminal Justice Information and Statistics Service.

Walsh, A. (1984). Differential sentencing patterns among felony sex offenders and nonsex offenders. Journal of criminal law and criminology, 75 (2), 443-458.

Wicker, A. W. (1975). An application of a multipletrait- multimethod logic to the reability of observational records. Personality and Social Psychology Bulletin, $4,575-579$. 


\section{Curriculo autores}

Miguel Angel Soria Verde.

Dr. En Psicología por la Universidad de Barcelona. Profesor de Psicología Criminal y Psicología Jurídica en la Licenciatura de Criminología de la Univ. Barcelona. Perito forense en el ámbito penal. Autor de diversos libros sobre el tema de la psicología criminal, judicial y jurídica. Aréas de interés: decisiones judiciales, violencia/ homicidio doméstico, mediación penal y psicología forense.

\section{Inmaculada Armadans Tremolosa.}

Dra. En Psicología por la Universidad de Barcelona. Profesora de Mediación y resolución de conflictos en la Univ. Barcelona. Profesora en la Escuela de Policía de Cataluña. Áreas de interés: mediación y resolución de conflictos, sistema judicial y medidas alternativas, trabajo policial.

Correspondencia: Miguel Angel Soria

Facultad de Psicología. Departamento de Psicología Social.

Universidad de Barcelona

Passeig Vall d'Hebron, 171

08035 Barcelona

E-mail: msoria@ub.edu

\section{Agradecimientos}

La investigación fue desarrollada gracias a la ayuda a la investigación concedida por el

Centre d'Estudis Jurídics i Formació Especialitzada del Departament de Justicia de la Generalitat de Catalunya. 